\title{
TENSORS, COLOURS, OCTAHEDRA
}

\author{
IMRE BÁRÁNY
}

\begin{abstract}
Several theorems in combinatorial convexity admit colourful versions. This survey describes old and new applications of two methods that can give such colourful results. One is the octahedral construction, the other is Sarkaria's tensor method.
\end{abstract}

\section{INTRODUCTION}

Theorems of Carathéodory, Helly, and Tverberg are classical results in combinatorial convexity. They all have coloured versions. Some others involve colours directly. For instance in Kirchberger's theorem [16], the elements of a finite set $X \subset \mathbb{R}^{d}$ are coloured Red and Blue, and the statement is that the Red and Blue points can be separated by a hyperplane if and only if for every $Y \subset X$ with $|Y| \leq d+2$, the Red and Blue points in $Y$ can be separated by a hyperplane.

The aim of this paper is to describe and explain old and new applications of two methods that have turned out to be useful when proving such colourful results. One is the octahedral construction, discovered and first used by László Lovász in 1991, which appeared in [4]. The other is Karinbir Sarkaria's tensor method, originally from [26] and developed further in [5].

In the next section Tverberg's theorem and its colourful version are presented. The octahedral construction is given in Section 3 with applications followed in later sections.

\section{TVERBERG's THEOREM AND ITS COLOURED VERSion}

Tverberg's theorem is a gem, one of my favourites. Here is what it says.

Theorem 2.1. Assume $d \geq 1, r \geq 2$ and $X \subset \mathbb{R}^{d}$ has $(r-1)(d+1)+1$ elements. Then $X$ has a partition into $r$ parts $X_{1}, \ldots, X_{r}$ such that $\bigcap_{1}^{r} \operatorname{conv} X_{i} \neq \emptyset$.

The number $(r-1)(d+1)+1$ is best possible here: for a general position $X$ with one fewer element, the affine hulls of an $r$-partition do not have a common point (by counting dimensions).

2000 Mathematics Subject Classification. Primary 52B22.

Key words and phrases. Theorems of Carathéodory and Tverberg, coloured versions, tensors, octahedra. 
The case $r=2$ is Radon's theorem from 1922 [22] that has a simple proof: Given $x \in \mathbb{R}^{d}$ we write $(x, 1)$ for the $(d+1)$-dimensional vector whose first $d$ components are equal to those of $x$, and the last one is 1. This time $|X|=d+2$ so the vectors $(x, 1) \in \mathbb{R}^{d+1}$ have a nontrivial linear dependence $\sum \alpha(x)(x, 1)=(0,0)$. Letting $X_{1}=\{x \in X$ : $\alpha(x) \geq 0\}$ and $X_{2}=\{x \in X: \alpha(x)<0\}$ is the partition needed. Indeed, defining $\alpha=\sum_{x \in X_{1}} \alpha(x)$ and $\alpha^{*}(x)=\alpha(x) / \alpha$ for $x \in X_{1}$ and $\alpha^{*}(x)=-\alpha(x) / \alpha$ for $x \in X_{2}$, we have convex combinations in

$$
z=\sum_{x \in X_{1}} \alpha^{*}(x) x=\sum_{x \in X_{2}} \alpha^{*}(x) x
$$

showing that $z \in \operatorname{conv} X_{1} \bigcap \operatorname{conv} X_{2}$.

There are several proofs of Tverberg's theorem, for instance in Tverberg [30, 31], Tverberg and Vrećica [32], Roudneff [24], Sarkaria [26], Bárány and Onn [5], Zvagelskii [35], none of them easy. We will give another proof in Section 8 which is from Arocha et al. [1].

The coloured version of Tverberg's theorem follows now.

Theorem 2.2. For every $d \geq 1$ and $r \geq 2$ there is $t=t(r, d)$ with the following property. Given sets $C_{1}, \ldots, C_{d+1} \in \mathbb{R}^{d}$ (called colours), each of size $t$, there are $r$ disjoint sets $S_{1}, \ldots, S_{r} \subset \bigcup_{1}^{d+1} C_{i}$ such that $\left|S_{j} \cap C_{i}\right|=1$ for every $i, j$ and $\bigcap_{1}^{r} \operatorname{conv} S_{j} \neq \emptyset$.

In other words, given colours $C_{1}, \ldots, C_{d+1} \subset \mathbb{R}^{d}$ of large enough size, there are $r$ disjoint and colourful sets $S_{j}$ whose convex hulls have a point in common. Colourful means that $S_{j}$ is a transversal of the $C_{i}$, that is, $S_{j}$ contains one element from each $C_{i}$. The need for this result emerged in connection with the halving plane problem (c.f. [3]). It was proved there that $t(3,2)$ is finite. Shortly afterward it was proved by Bárány and Larman [4] that $t(r, 2)=r$ for all $r$, clearly the best possible result. The same paper presents Lovász's proof that $t(2, d)=2$ for all $d$, the first application of the octahedral method. To simplify notation we write $[k]$ for the set $\{1,2, \ldots, k\}$.

\section{The octahedral CONSTRUCTION}

Proof of $t(2, d)=2$. We have $C_{i}=\left\{a_{i}, b_{i}\right\} \subset \mathbb{R}^{d}, i \in[d+1]$. Note that we may exchange the names of $a_{i}$ and $b_{i}$ later. We want to choose a transversal $T$ from $C_{1}, \ldots, C_{d+1}$ such that the convex hulls of $T$ and of the complementary transversal $\bar{T}$ have a point in common. For this purpose let

$$
Q^{d+1}=\operatorname{conv}\left\{ \pm e_{1}, \ldots, \pm e_{d+1}\right\}
$$

be the standard octahedron in $\mathbb{R}^{d+1}$ (the $e_{i}$ are the usual basis vectors). We define a map $f: \partial Q^{d+1} \rightarrow \mathbb{R}^{d}$ by setting $f\left(e_{i}\right)=a_{i}$ and $f\left(-e_{i}\right)=b_{i}$, and then extend $f$ simplicially to $\partial Q^{d+1}$, that is, to the facets of $Q^{d+1}$. Note that $f$ maps the facets of $Q^{d+1}$ to the convex hull of a transversal 
$T$ exactly, and the opposite facet is mapped to $\operatorname{conv} \bar{T}$. So what we need is a pair of opposite facets whose images intersect.

This cries out for the Borsuk-Ulam theorem: $\partial Q^{d+1}$ is homeomorphic to $S^{d}$ and so $f$ is an $S^{d} \rightarrow \mathbb{R}^{d}$ map. By a variant of Borsuk-Ulam there are antipodal points $z,-z \in \partial Q^{d+1}$ with $f(z)=f(-z)$. If $z$ lies on a facet $F$, then $-z$ lies on the opposite facet $\bar{F}$. For simpler writing assume that $F=\operatorname{conv}\left\{e_{1}, \ldots, e_{d+1}\right\}$, then $\bar{F}=\operatorname{conv}\left\{-e_{1}, \ldots,-e_{d+1}\right\}$, and we see that conv $\left\{a_{1}, \ldots, a_{d+1}\right\}$ and conv $\left\{b_{1}, \ldots, b_{d+1}\right\}$ have $f(z)=$ $f(-z)$ as a common point.

Actually, more is true: if $z=\sum_{1}^{d+1} \gamma_{i} e_{i}$, then $-z=\sum_{1}^{d+1} \gamma_{i}\left(-e_{i}\right)$, and the common point is $\sum_{1}^{d+1} \gamma_{i} a_{i}=\sum_{1}^{d+1} \gamma_{i} b_{i}$. Thus the common point comes with the same coefficients in the convex combinations.

This is the octahedral method. The basic idea is that facets of the octahedron correspond to transversals of $C_{1}, \ldots, C_{d+1}$, transversals have the structure of $\partial Q^{d+1}$, and disjoint transversals come from opposite facets, and the next step is the use of algebraic topology like the BorsukUlam theorem above.

Unfortunately the method does not work for $r \geq 3$. It was conjectured in [4] that $t(r, d)=r$ for all $r$ and $d$. Finiteness of $t(r, d)$ was proved by Živaljević and Vrećica [34] using equivariant topology. Their result is that $t(r, d) \leq 2 r-1$ if $r$ is a prime (which implies finiteness of $t(r, d)$ for all $r$ ). The same was proved by different methods by Björner et al. [8] and by Matoušek [18]. More recently Blagojević, Matschke, and Ziegler [9] showed that $t(r, d)=r$ if $r+1$ is a prime which is again best possible. The strange primality condition in all cases is needed because cyclic groups of prime order behave better in equivariant topology. But the theorem is probably true for every $r$, the primality condition is required for the method and not for the problem. It is however disappointing (for a convex geometer) that a completely convex (or linear, if you wish) problem does not have a direct convex (or linear) proof, and topology seems a necessity here. Finding a purely geometric proof remains a challenge. The interested reader may wish to read Günter Ziegler's fascinating article [33] about Tverberg's theorem and its colourful version.

Remark. Quite recently, Pablo Soberón [27] has found another (and simpler) proof of $t(2, d)=2$. It starts with the observation that the vectors $a_{i}-b_{i}, i \in[d+1]$ are linearly dependent, so $\sum_{1}^{d+1} \gamma_{i}\left(a_{i}-b_{i}\right)=0$ for some not all zero $\gamma_{i}$. Some $\gamma_{i}$ may be negative, but then we exchange the names of $a_{i}$ and $b_{i}$ which makes $\gamma_{i}$ positive. Then $\sum \gamma_{i} a_{i}=\sum \gamma_{i} b_{i}$. Assuming as we can that $\sum \gamma_{i}=1, \sum \gamma_{i} a_{i}=\sum \gamma_{i} b_{i}$ is a common point of the convex hulls of transversals $a_{1}, \ldots, a_{d+1}$ and $b_{1}, \ldots, b_{d+1}$. Note that even the coefficients are the same. So this method gives exactly the same result as the octahedral construction. A little extra 
is the efficient algorithm that follows from this proof. The paper [27] gives precise conditions for the existence of colourful partitions whose convex hulls have a common point with equal coefficients. The proof uses tensors as in Sarkaria's lemma which will be described in Section 7.

\section{Colourful Carathéodory theorem}

Carathéodory's classical theorem says in essence that being in the convex hull has a very finite reason. Precisely, if $A \subset \mathbb{R}^{d}$ and $a \in$ conv $A$, then $a \in \operatorname{conv} B$ for some $B \subset A$ with $|B| \leq d+1$. The colourful version of this theorem is an old result of mine [2].

Theorem 4.1. If $A_{1}, \ldots, A_{d+1} \subset \mathbb{R}^{d}$ and $a \in \bigcap_{1}^{d+1}$ conv $A_{i}$, then there is a transversal $a_{i} \in A_{i}$ for all $i$, such that $a \in \operatorname{conv}\left\{a_{1}, \ldots, a_{d+1}\right\}$.

The colourful version contains the original one: simply take $A_{i}=A$ for every $A$. A natural question is how many such transversals are there, and the natural setting for the question is when $a$ is the origin (which makes no difference), the points in $\bigcup_{1}^{d+1} A_{i}$ together with the origin are in general position, and each $A_{i}$ has exactly $d+1$ elements. Of course, $0 \notin \bigcup_{1}^{d+1} A_{i}$, and we may assume that each $A_{i} \subset S^{d-1}$, the unit sphere of $\mathbb{R}^{d}$. We call a transversal $\left\{a_{1}, \ldots, a_{d+1}\right\}$ special if the origin lies in its convex hull. Define $\tau(d)$ as the minimal number of special transversals under these conditions.

A neat construction from Deza et al. [11] shows that $\tau(d) \leq d^{2}+1$ and it is not hard to check that $\tau(2)=5$. Carathéodory's theorem has a cone hull or positive hull version, slightly stronger than the convex one. We write pos $A$ for the cone hull of the elements in $A \subset \mathbb{R}^{d}$, that is, pos $A$ is the set of vectors $\sum_{1}^{n} \gamma_{i} a_{i}$ with $\gamma_{i} \geq 0$ and $a_{i} \in A$ for all $i \in[n]$ and all $n \in \mathbb{Z}$.

Theorem 4.2. If $A_{1}, \ldots, A_{d} \subset \mathbb{R}^{d}$ and $a \in \bigcap_{1}^{d}$ pos $A_{i}$, then there is a transversal $a_{i} \in A_{i}$ for all $i$ such that $a \in \operatorname{pos}\left\{a_{1}, \ldots, a_{d}\right\}$.

It is quite easy to check (or see [2] for the proof) that this result has the following consequence.

Corollary 4.3. If $a \in \mathbb{R}^{d}, A_{1}, \ldots, A_{d} \subset \mathbb{R}^{d}$, and $0 \in \bigcup_{1}^{d}$ conv $A_{i}$, then there is a transversal $a_{i} \in A_{i}$ for all $i$ such that $0 \in \operatorname{conv}\left\{a, a_{1}, \ldots, a_{d}\right\}$.

The Corollary shows immediately that every point in $\bigcup_{1}^{d+1} A_{i}$ belongs to at least one special transversal, so $\tau(d) \geq d+1$. The octahedral construction has been used to improve this bound to a quadratic one, in several papers. In particular, Bárány and Matoušek [6] show $\tau(d) \geq d(d+1) / 5$ and $\tau(3)=10$ (which is best possible), Stephen and Thomas [29] prove $\tau(d) \geq(d+2)^{2} / 4$, and Deza et al. [12] give $\tau(d) \geq(d+1)^{2} / 2$, which is further improved to $\tau(d) \geq \frac{1}{2} d^{2}+\frac{7}{2} d-8$ when $d \geq 4$ by Deza, Meunier, and Sarrabezolles in [13]. 
How can the octahedral construction help here? Well, it is clear that $a_{1}, \ldots, a_{d+1}$ is a special transversal iff $-a_{d+1} \in \operatorname{pos}\left\{a_{1}, \ldots, a_{d}\right\}$. Fix now the special transversal $a_{1}, \ldots, a_{d+1}$ and consider a partial transversal $b_{1} \in A_{1}, \ldots, b_{d} \in A_{d}$ with $b_{i}$ different from $a_{i}$ for all $i$. The octahedral construction defines a map $f: \partial Q^{d} \rightarrow \mathbb{R}^{d}$ by setting $f\left(e_{i}\right)=a_{i}, f\left(-e_{i}\right)=b_{i}$ (for all $i$ ), and then extend it simplicially to $\partial Q^{d}$. As $0 \notin f\left(\partial Q^{d}\right)$ by the general position assumption, we can define $g(x)=f(x) /\|f(x)\|$. The map $g: \partial Q^{d} \rightarrow S^{d-1}$ is continuous and is essentially an $S^{d-1} \rightarrow S^{d-1}$ map, so if it takes some (non-critical) value, then it takes it at least twice, or else it takes every value at least once.

More precisely, if the degree of $g$ is nonzero, then $g$ takes every value in $S^{d-1}$ at least once, and if its degree is zero, then it takes every non-critical (in the sense of Sard's Lemma, see Milnor's book [19]) value at least twice. But $g$ takes the value $-a_{d+1}$ at least once, since $-a_{d+1} \in g\left(\partial Q^{d}\right)$. Moreover, this value is non-critical because of the general position assumption. Writing $T=\left\{a_{1}, \ldots, a_{d+1}\right\}$ and $B=\left\{b_{1}, \ldots, b_{d}\right\}$ we have established the following fact.

Lemma 4.4. Under the above condition either $T \cup B$ contains another special transversal, different from $T$, or every $b_{d+1} \in A_{d+1} \backslash\left\{a_{d+1}\right\}$ belongs to a special transversal from $T \cup B$.

This consequence of the octahedral construction is used, with varying outcome, in all quadratic lower bounds to $\tau(d)$. But the lemma also leads to a completely combinatorial problem: determine the minimum number of edges a hypergraph $\mathcal{H}$ can have provided it is a $(d+1)$ partite $(d+1)$-uniform hypergraph with partition classes $A_{1}, \ldots, A_{d+1}$, $\left|A_{i}\right|=d+1$ for each $i$, and satisfies the following conditions (mimicking those of the special transversals):

- for every $a \in \bigcup_{1}^{d+1} A_{i}$ there is $T \in \mathcal{H}$ with $a \in T$

- for every $i$ and for every $T \in \mathcal{H}$ with $T \cap A_{i}=a_{i}$, and for every $B=\left\{b_{1}, \ldots, b_{i-1}, b_{i+1}, \ldots, b_{d+1}\right\}$ with $B$ disjoint from $T$, either there is $T^{*} \in \mathcal{H}, T^{*} \neq T$ with $T^{*} \subset T \cup B$, or for every $a \in A_{i}$ there is $T^{*} \in \mathcal{H}$ with $a \in T^{*}$ and $T^{*} \backslash\{a\} \subset T \cup B$.

Here the first condition comes from Corollary 4.3, and the second from Lemma 4.4 as the role of $a_{d+1}$ can be taken be an arbitrary $a \in$ $\bigcup A_{i}$. Note however that the condition $0 \in \operatorname{conv} A_{i}$ is lost in this combinatorial setting.

Open question 4.5. For a hypergraph $\mathcal{H}$ with these properties, does $|\mathcal{H}|$ have to have at least $d^{2}+1$ edges? ${ }^{1}$ Even with no hypergraph, is it true that $\tau(d)=d^{2}+1$ ?

\footnotetext{
${ }^{1}$ This question has recently been settled in the affirmative by Pauline Sarrabezolles [25], implying $\tau(d)=d^{2}+1$.
} 


\section{Colourful Carathéodory strengthened}

The following result is a generalization of Theorem 4.1. It was found at the same time on two different continents, and was published by Holmsen, Pach, Tverberg [15] and by Arocha, Bárány, Fabila, Bracho, Montejano [1]. The proof is based on the octahedral construction. In both cases the original target was a colourful Helly type theorem on the sphere, see [15] or [1].

Theorem 5.1. If $A_{1}, \ldots, A_{d+1} \subset \mathbb{R}^{d}$, none of them empty and $a \in$ conv $\left(A_{i} \cup A_{j}\right)$ for all distinct $i, j \in[d+1]$, then there is a transversal $a_{i} \in A_{i}$ for all $i$, such that $a \in \operatorname{conv}\left\{a_{1}, \ldots, a_{d+1}\right\}$.

Proof. We identify $a$ with the origin. We can assume that every $A_{i}$ is finite. Let $T$ be the transversal with $a_{i} \in A_{i}$ for $i \in[d+1]$ whose convex hull $\triangle=\operatorname{conv} T$ is closest to the origin. Let $z \in \triangle$ be this closest point. If $z=0$ we are done, so assume $z \neq 0$, and let $H$ be the hyperplane passing through, and orthogonal to, z. Write $H^{+}$for the closed halfspace bounded by $H$ and not containing 0 . As $z$ is on the boundary of the simplex $\triangle$, it is in the convex hull of a proper subset of $T$, say of $\left\{a_{1}, \ldots, a_{d}\right\}$.

We claim that $z$ lies in the relative interior of conv $\left\{a_{1}, \ldots, a_{d}\right\}$. Assume on the contrary that $z$ is in the convex hull of $\left\{a_{1}, \ldots, a_{d-1}\right\}$, say. There is a point $b \in\left(A_{d} \cup A_{d+1}\right) \backslash H^{+}$as otherwise $A_{d} \cup A_{d+1} \subset H^{+}$so their convex hull does not contain the origin, contrary to the condition of the theorem. Now $\left\{a_{1}, \ldots, a_{d-1}, b\right\}$ can be extended to a transversal $T^{*}$ whose convex hull of contains the segment $[z, b]$. But $[z, b]$, and consequently conv $T^{*}$, contains a point closer to the origin than $z$, contradicting the choice of $T$.

Note that $A_{d+1} \subset H^{+}$since replacing $a_{d+1}$ by any $b \in A_{d+1} \backslash H^{+}$ would give a transversal whose convex hull is closer to the origin than conv $T$. Let $H_{0}$ be the hyperplane parallel to $H$ and containing the origin, and $H^{-}$be the closed halfspace bounded by $H_{0}$ and not containing $\triangle$.

It follows that there is $b_{i} \in A_{i} \cap H^{-}$for every $i \in[d]$, as otherwise $A_{i} \cup A_{d+1}$ lies in the complement of $H^{-}$and is then separated from the origin. We can apply the octahedral construction now. Define $f: \partial Q^{d} \rightarrow \mathbb{R}^{d}$ by setting $f\left(e_{i}\right)=a_{i}, f\left(-e_{i}\right)=b_{i}$ and extend $f$ simplicially to the facets of $Q^{d}$.

Again, $\partial Q^{d}$ is an $S^{d-1}$ so removing $f\left(\partial Q^{d}\right)$ from $\mathbb{R}^{d}$ results in an unbounded connected component and finitely many bounded connected components (by the Jordan curve theorem in higher dimensions). The unbounded component contains the interior of $H^{+}$. The segment $[0, z)$ is disjoint from $f\left(\partial Q^{d}\right)$ so it lies in some connected component $\Omega$. It is clear that $\Omega$ is not the unbounded connected component.

Consider now a point $a \in A_{d+1}$, and the halfline $L$ starting at 0 in direction $-a$. $L$ starts in $\Omega$ and ends up in the unbounded component. 


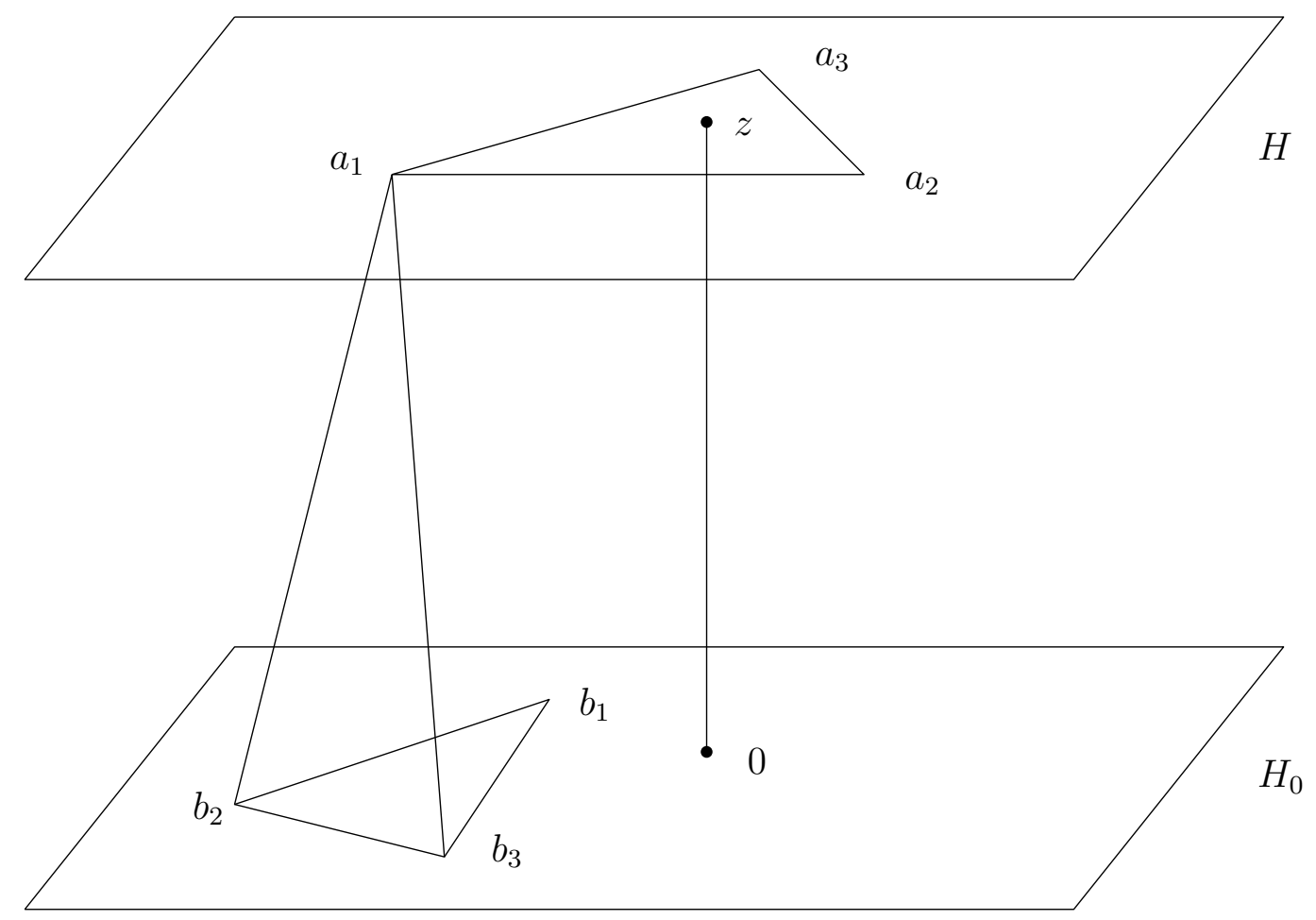

FiguRE 1. Figure for Theorem 6.1, almost works for Theorem 5.1. Only parts of $f\left(\partial Q^{d}\right)$ are drawn.

So it must intersect $f\left(\partial Q^{d}\right)$ at some point $v=L \cap f(F)$ where $F$ is a facet of $\partial Q^{d}$. Then $v$ is in the convex hull of a transversal of $A_{1}, \ldots, A_{d}$ (even of $\left\{a_{1}, b_{1}\right\}, \ldots,\left\{a_{d+1}, b_{d+1}\right\}$ ). Since $0 \in[a, v]$, the convex hull of this partial transversal and $a$ contains the origin, contrary to the choice of $T$.

The theorem has $d(d+1) / 2$ conditions, one for each pair $i, j$. All of them are needed as the following example shows. Assume the points $a, x_{1}, \ldots, x_{d+1}, y$ are in general position and $a \in \operatorname{conv}\left\{x_{1}, \ldots, x_{d+1}\right\}$, and let $A_{i}=\left\{x_{1}, \ldots, x_{d+1}\right\}$ for $i \in[d-1]$ and $A_{d}=A_{d+1}=\{y\}$. There is no transversal whose convex hull would contain $a$ yet for every pair $i, j$ apart from $d, d+1, a \in \operatorname{conv}\left(A_{i} \cup A_{j}\right)$. The same example shows that the conditions $a \in \operatorname{conv}\left(A_{i} \cup A_{j} \cup A_{k}\right)$ for every triple $i, j, k$ do not work. More disappointingly, the result does not extend to the cone hull, as shown by a very simple example in $\mathbb{R}^{2}$.

Open question 5.2. It would be interesting to design an effective algorithm that, under the conditions of Theorem 4.1, finds a colourful simplex whose convex hull contains the origin. The proof of Theorem 4.1, and also that of Theorem 5.1 only gives the existence of such a simplex. So in fixed dimension they give an algorithm with at most 
$(d+1)^{d+1}$ steps, which is way too many when dimension is part of the input. For further information about this question see Bárány, Onn [5].

\section{Colourful Carathéodory for connected compacta}

A theorem of Fenchel [14] asserts that if a compact set $A \subset \mathbb{R}^{d}$ is connected, then $a \in \operatorname{conv} A$ implies the existence of $B \subset A$ with $a \in$ conv $B$ and $|B| \leq d$. So the Carathéodory number can be lowered. The colourful version of Fenchel's result is given in Bárány and Karasev [7]. Its proof is based on the octahedral construction, again. Recall that if $A \subset \mathbb{R}^{d}$ is connected and compact, then for every hyperplane $H$ disjoint from $A$, one of the open halfspaces bounded by $H$ contains $A$.

Theorem 6.1. Assume $A_{1}, \ldots, A_{d} \subset \mathbb{R}^{d}$ are compact connected sets with $0 \in \bigcap_{1}^{d}$ conv $A_{i}$. Then there is a transversal $a_{i} \in A_{i}(i \in[d]$ such that $0 \in \operatorname{conv}\left\{a_{1}, \ldots, a_{d}\right\}$.

The proof is similar to the previous one. Choose a transversal $T=$ $\left\{a_{1}, \ldots, a_{d}\right\}$ whose convex hull $\triangle$ is closest to the origin, and let $z \in \triangle$ be this closest point. If $z=0$ we are done, so suppose $z \neq 0$. It is easy to see (we omit the details) that $\triangle$ is a $d$-1-dimensional simplex and that $z$ lies in the relative interior of $S$.

Let $H$ be the hyperplane passing through, and orthogonal to, $z$, clearly $\triangle \subset H$. Again, let $H_{0}$ be the hyperplane parallel to $H$ and containing the origin. As $A_{i}$ is connected, there is a point $b_{i} \in H_{0} \cap A_{i}$ for every $i \in[d]$. The octahedral construction applies the same way as before. So we have $f: \partial Q^{d} \rightarrow \mathbb{R}^{d}$, the simplicial extension from the vertices $f\left(e_{i}\right)=a_{i}, f\left(-e_{i}\right)=b_{i}$. This time $f\left(\partial Q^{d}\right)$ lies between $H$ and $H_{0}$, and evidently $0 \notin f\left(\partial Q^{d}\right)$. Again, removal of $f\left(\partial Q^{d}\right)$ from $\mathbb{R}^{d}$ yields connected components, and 0 is in the unbounded one. But the points on the segment $[0, z)$, close enough to $z$ lie in a bounded component. This shows that the open segment $(0, z)$ intersects $f\left(\partial Q^{d}\right)$. The intersection point is in the convex hull of a transversal and closer to the origin than $z$. Contradiction.

In [7] a second (and interesting) proof of the theorem is given which uses vector bundles and has some further consequences.

\section{SARKARIA'S LEMMA}

Assume $X_{1}, X_{2}, \ldots, X_{r} \subset \mathbb{R}^{d}$ are finite sets, $r \geq 2$. There is a good necessary and sufficient condition for $\bigcap_{1}^{r} \operatorname{conv} X_{i}=\emptyset$ which we now describe.

Theorem 7.1. Under the above conditions, $\bigcap_{1}^{r} \operatorname{conv} X_{i}=\emptyset$ if and only if there are closed halfspaces $D_{1}, \ldots, D_{r}$ with conv $X_{i} \subset D_{i}$ for every $i \in[r]$ such that $\bigcap_{1}^{r} D_{i}=\emptyset$. 
The proof is easy. One direction is trivial. For the other one set $K_{i}=$ conv $X_{i}$. The case $r=2$ is just the separation theorem for convex sets. For larger $r$ we have $K_{1} \cap \bigcap_{2}^{r} K_{i}=\emptyset$ so by separation there is a closed halfspace $D_{1}$ containing $K_{1}$ with $D_{1} \cap \bigcap_{2}^{r} K_{i}=\emptyset$. This way $K_{1}$ is replaced by $D_{1}$, and the same way $K_{2}$ is replaced by $D_{2}$, etc. After step $j-1$ we have $\bigcap_{1}^{j-1} D_{i} \cap \bigcap_{j}^{r} K_{i}=\emptyset$ and so $K_{j} \cap\left(\bigcap_{1}^{j-1} D_{i} \cap \bigcap_{j+1}^{r} K_{i}\right)=\emptyset$. Here $K_{j}$ is convex, compact and $\bigcap_{1}^{j-1} D_{i} \cap \bigcap_{j+1}^{r} K_{i}$ is convex so the separation theorem applies.

The case when $\bigcap_{1}^{r} D_{i}=\emptyset$ can be characterized by duality. Assume $D_{i}=\left\{x \in \mathbb{R}^{d}: a_{i} x \leq \alpha_{i}\right\}$.

Theorem 7.2. With the above notation $\bigcap_{1}^{r} D_{i}=\emptyset$ if and only if $(0,-1) \in \operatorname{pos}\left\{\left(a_{i}, \alpha_{i}\right): i \in[r]\right\}$.

Sketch of proof. The condition $\bigcap_{1}^{r} D_{i}=\emptyset$ is equivalent to "the system of linear inequalities $a_{i} x \leq \alpha_{i}, i \in[r]$ has no solution". Then Farkas's lemma proves the theorem.

This an outer or dual type characterization. Sarkaria's lemma is an inner characterization of the fact that $\bigcap_{1}^{k}$ conv $X_{i}=\emptyset$. We need an artificial tool: choose vectors $v_{1}, \ldots, v_{r} \in \mathbb{R}^{r-1}$ so that their unique (up to a multiplier) linear dependence is $v_{1}+\cdots+v_{r}=0$. Suppose that $X_{1}, X_{2}, \ldots, X_{r} \subset \mathbb{R}^{d}$ are finite sets, and write $X=\bigcup_{1}^{r} X_{i}$. We assume further that the $X_{i}$ s are disjoint. (Alternatively, we can consider $X$ a multiset.) Each $x \in X$ comes from a unique $X_{i}=X_{i(x)}$. With each such $x$ we associate the tensor

$$
\bar{x}=v_{i} \otimes(x, 1) \in \mathbb{R}^{n}
$$

where $n=(r-1)(d+1)$. The tensor $\bar{x}$ can be thought of as an $(r-1)$ by $(d+1)$ matrix as well. Here is Sarkaria's lemma [26], in the form given in Bárány and Onn [5]. (Originally it used number fields instead of tensors.)

Theorem 7.3. Under the above conditions, $\bigcap_{1}^{r} \operatorname{conv} X_{i}=\emptyset$ if and only if $0 \notin \operatorname{conv} \bar{X}$.

Proof. We prove the theorem by showing that $0 \in \operatorname{conv} \bar{X}$ iff $\bigcap_{1}^{r} \operatorname{conv} X_{i} \neq \emptyset$.

If $0 \in \operatorname{conv} \bar{X}$, then there are $\alpha(x) \geq 0$ for all $x \in X$ such that

$$
\sum_{x \in X} \alpha(x)=1 \text { and } \sum_{x \in X} \alpha(x) \bar{x}=0 .
$$


Replacing $\bar{x}$ by $v_{i} \otimes(x, 1)$ gives

$$
\begin{aligned}
0 & =\sum_{x \in X} \alpha(x) \bar{x}=\sum_{i=1}^{r} \sum_{x \in X_{i}} \alpha(x) v_{i} \otimes(x, 1) \\
& =\sum_{i=1}^{r} v_{i} \otimes \sum_{x \in X_{i}} \alpha(x)(x, 1) .
\end{aligned}
$$

Set $z_{i}=\sum_{x \in X_{i}} \alpha(x)(x, 1) \in \mathbb{R}^{d+1}$ for $i \in[r]$. We claim that $z_{1}=$ $z_{2}=\cdots=z_{r}$. By symmetry it suffices to show that $z_{1}=z_{2}$. By the choice of the vectors $v_{1}, \ldots, v_{r}$ there is $u \in \mathbb{R}^{r-1}$ such that $u v_{1}=$ $1, u v_{2}=-1$ and $u v_{i}=0$ for all $i>2$. Multiplying the last formula by $u$ from the left gives $0=\sum_{i=1}^{r} u v_{i} \otimes z_{i}=z_{1}-z_{2}$.

This implies, in particular, that the last coordinate of each $z_{i}$ is equal to $1 / r$. Thus $y_{i}=\sum_{x \in X_{i}} r \alpha(x) x$ is a convex combination of the elements of $X_{i}$, and $y=y_{1}=\cdots=y_{r}$. Consequently $y$ is a common element of each conv $X_{i}$.

The steps of this proof can be reversed easily showing that condition $\bigcap_{1}^{r} \operatorname{conv} X_{i} \neq \emptyset$ implies $0 \in \operatorname{conv} \bar{X}$.

Remark. Note that when $r=2, v_{1}=1$ and $v_{2}=-1$, Sarkaria's lemma gives $X_{1}$ resp. $X_{2}$ as the set of elements with positive (and negative) coefficients in the linear dependence of $\left(x_{1}, 1\right), \ldots,\left(x_{d+2}, 1\right)$. Sarkaria's tensor method is a direct and beautiful generalization of the proof of Radon's theorem.

\section{KirChBERGER GENERALIZED}

Recall Kirchberger's theorem [16] from the first section with Red and Blue points. We want to have more colours this time, so we give the theorem in a slightly different form. The elements of a finite sets $X \subset$ $\mathbb{R}^{d}$ are coloured Red or Blue which is simply a partition of $X$ into $X_{1}$, (the Red points) and $X_{2}$, (the Blue ones). We say that $X$ is separated along the colours if conv $X_{1} \cap$ conv $X_{2}=\emptyset$. Now Kirchberger's theorem says that $X$ is separated along the colours iff every subset of $X$, of size at most $d+2$, is separated along the colours.

The extension to more colours is quite natural now. Assume a finite set (or multiset) $X \subset \mathbb{R}^{d}$ is coloured with $r \geq 2$ colours, that is, there is a partition $X=X_{1} \cup \cdots \cup X_{r}$. We say that $X$ is separated along the colours if $\bigcap_{1}^{r}$ conv $X_{i}=\emptyset$. The colourful version of Kirchberger's theorem is a result of A. Pór [21]:

Theorem 8.1. With the above notation $X \subset \mathbb{R}^{d}$ is separated along the colours if and only if every subset of $X$ of size at least $(r-1)(d+1)+1$ is separated along the colours.

Note that $r=2$ is the original Kirchberger theorem. Theorem 8.1 can be proved using Theorem 7.2, for instance. But here we aim for 
more. Set $n=(r-1)(d+1)$ and assume that, for every $i \in[r]$ and for every $j \in[n+1]$, a finite set $X_{i, j} \subset \mathbb{R}^{d}$ is given (which may be empty). This can be thought of as an $r$ by $(n+1)$ matrix whose $i, j$-entry is the set $X_{i, j}$.

$$
\begin{array}{ccccc} 
& G_{1} & G_{2} & \ldots & G_{n+1} \\
X_{1} & X_{1,1} & X_{1,2} & \ldots & X_{1, n+1} \\
X_{2} & X_{2,1} & X_{2,2} & \ldots & X_{2, n+1} \\
\vdots & \vdots & \vdots & \vdots & \vdots \\
X_{r} & X_{r, 1} & X_{r, 2} & \ldots & X_{r, n+1}
\end{array}
$$

We call the sets $X_{i}=\bigcup_{j=1}^{n+1} X_{i, j}$ colours and the sets $G_{j}=\bigcup_{i=1}^{r} X_{i, j}$ groups, (nothing to do with groups in algebra though). A transversal of this system is a set $Y=\left\{y_{1}, y_{2}, \ldots, y_{n+1}\right\}$ if $y_{j} \in G_{j}$ for every $j$. In the multiset case, of course, every $y_{j} \in Y$ comes from a uniquely determined $X_{i, j} \subset G_{j}$. The following result is form Arocha et al. [1].

Theorem 8.2. Under the above conditions, if every transversal is separated along the colours, then so is some group $G_{j}$.

Proof. We use Sarkaria's lemma. A transversal $Y$ is separated along the colours iff $0 \notin \operatorname{conv} \bar{Y}$ (where $\bar{Y} \in \mathbb{R}^{n}$ ). If $0 \notin \operatorname{conv} \bar{Y}$ for all transversals, then by Theorem 4.1 , we can't have $0 \in \operatorname{conv} \overline{G_{j}}$ for all $j$, meaning that $0 \notin \operatorname{conv} \overline{G_{j}}$ for some $j \in[n+1]$. Then, by Sarkaria's lemma again, $G_{j}$ is separated along the colours.

Note that using Theorem 5.1 instead of colourful Carathéodory, one gets a little more, namely, two groups whose union is separated along the colours.

We give two applications of this result. The first is the colourful Kirchberger Theorem 8.1. The finite $X$ is partitioned as $X_{1} \cup \cdots \cup X_{r}$ and we define $X_{i, j}=X_{i}$ for all $j=[n+1]$. A transversal $Y$ in this case is sequence of $n+1$ elements of $X$ (possibly with repetitions), and $Y$ is separated along the colours simply means that $\bigcap_{1}^{r} \operatorname{conv}\left(Y \cap X_{i}\right)=\emptyset$. If all transversals are separated along the colours, then so is one group by the theorem we just proved. But all groups are the same, which means that $X_{1}, \ldots, X_{r}$ are separated along the colours.

The second application is a proof of Tverberg's theorem. We are given a set $X=\left\{x_{1}, \ldots, x_{n+1}\right\}$ in $\mathbb{R}^{d}, n=(r-1)(d+1)$ and we are going to find an $r$-partition $X_{1} \cup \cdots \cup X_{r}$ of $X$ with $\bigcap_{1}^{r} \operatorname{conv} X_{i} \neq \emptyset$. Define $X_{i, j}=\left\{x_{j}\right\}$ for all $i \in[r]$. As each group is a single point repeated $r$ times, no group is separated along the colours. Theorem 8.2 implies then that some transversal, say $Y$, is not separated along the colours. Note that each $y_{j} \in Y$ comes from a unique $X_{i, j}$. For a fixed $i$, let $X_{i}$ be the set of $y_{j} \in Y$ that come from $X_{i, j}$. This is a partition of $X$. The fact that $Y$ is not separated along the colours means exactly that $\bigcap_{1}^{r} \operatorname{conv} X_{i} \neq \emptyset$, as required. 
Open question 8.3. Give an effective algorithm to find a Tverberg partition of a set $X \subset \mathbb{R}^{d}$ with $(r-1)(d+1)+1$ elements. Note that a positive answer to Open question 4.5 would solve this problem, via Sarkaria's lemma.

\section{TVERBERG'S THEOREM With TOLERANCE}

A partition of a finite set $X \subset \mathbb{R}^{d}$ with parts $X_{1}, \ldots, X_{r}$ has tolerance $t$ if for every set $T \subset X$ of size $t$

$$
\bigcap_{1}^{r} \operatorname{conv}\left(X_{i} \backslash T\right) \neq \emptyset .
$$

A partition with tolerance with $t=0$ is just a Tverberg partition. The question is what size of $X$, as a function of $d, r$ and $t$, guarantees the existence of an $r$-partition with tolerance $t$. This question is open even in the case $r=2, t=1$ (see [28] for more information). Recently Soberón and Strausz [28] have given an upper bound on this number. Their argument uses Sarkaria's lemma, that is why we present it here.

Theorem 9.1. Suppose $d \geq 1, r \geq 2, t \geq 0$ are integers. Every $X \subset \mathbb{R}^{d}$ with at least $(r-1)(d+1)(t+1)+1$ elements has a partition into $r$ parts with tolerance $t$.

Note that the slightly weaker bound $(t+1)[(r-1)(d+1)+1]$ follows from Tverberg's theorem directly.

It will be convenient to say that $S \subset \mathbb{R}^{d}$ captures the origin if $0 \in$ conv $S$, and $S$ captures the origin with tolerance $t$ if $0 \in \operatorname{conv}(S \backslash T)$ for every $T \subset S$ with $|T| \leq t$.

We need a definition and a lemma. Given $S \subset S^{\prime} \subset \mathbb{R}^{p}$ and a group $G$, an action of $G$ on $S^{\prime}$ is said to be compatible with $S$ if the following holds:

- If $A \subset S^{\prime}$ captures the origin, then so does $g A$ for every $g \in G$,

- $G a$ captures the origin for every $a \in S$.

Lemma 9.2. Assume $p \geq 1$ and $t \geq 0$ are integers, $n=p(t+1)+1$, $S=\left\{a_{1}, \ldots, a_{n}\right\} \subset R^{d}$, and $G$ is a finite group with $|G| \leq p$. If there is an action of $G$ on a set $S^{\prime}$ which is compatible with $S \subset S^{\prime}$, then there are $g_{j} \in G$ (for all $j \in[n]$ ) such that the set $\left\{g_{1} a_{1}, \ldots, g_{n} a_{n}\right\}$ captures the origin with tolerance $t$.

We prove the theorem first.

Proof of Theorem 9.1 Set $n=(r-1)(d+1)(t+1)+1, X=$ $\left\{x_{1}, \ldots, x_{n}\right\}, p=(r-1)(d+1)$, and let $v_{1}, v_{2}, \ldots, v_{r} \in \mathbb{R}^{r-1}$ be the vertices of a regular simplex centered at the origin. So $\alpha_{1} v_{1}+\cdots+$ 
$\alpha_{r} v_{r}=0$ iff $\alpha_{1}=\cdots=\alpha_{r}$. Then $v_{1}, \ldots, v_{r}$ satisfy the conditions of Sarkaria's lemma. Define

$$
\begin{aligned}
S^{\prime} & =\left\{v_{i} \otimes\left(x_{j}, 1\right) \in \mathbb{R}^{p}: i \in[r], j \in[n]\right\} \text { and } \\
S & =\left\{v_{r} \otimes\left(x_{j}, 1\right) \in \mathbb{R}^{p}: j \in[n]\right\} .
\end{aligned}
$$

There is a natural action of $\mathbb{Z}_{r}$ (the cyclic group of order $r$ ) on $S^{\prime}$, given by $m\left(v_{i} \otimes\left(x_{j}, 1\right)\right)=v_{i+m} \otimes\left(x_{j}, 1\right)$ where $i+m$ is taken $\bmod r$.

Next we check the conditions of Lemma 9.2. For each $a \in S^{\prime}, \mathbb{Z}_{r} a$ captures the origin as $\sum_{1}^{r} v_{i}=0$. Suppose $A$ is a subset of $S^{\prime}$ that captures the origin. As the simplex with vertices $v_{1}, \ldots, v_{r}$ is regular, the coefficients of the convex combination that give 0 for $A$ work for $g A$ to give 0 again for every $g \in \mathbb{Z}_{r}$. Note that the condition $d \geq 1$ is needed as it implies $p \geq r=|G|$.

So the lemma applies and gives $m_{j} \in \mathbb{Z}_{k},(j \in[n])$ such that, with $m_{j}\left(v_{r} \otimes\left(x_{j}, 1\right)\right)=v_{m_{j}} \otimes\left(x_{j}, 1\right)$ the set

$$
\begin{aligned}
Y & =\left\{m _ { 1 } \left(v_{r} \otimes\left(x_{1}, 1\right), m_{2}\left(v_{r} \otimes\left(x_{2}, 1\right), \ldots, m_{n}\left(v_{r} \otimes\left(x_{n}, 1\right)\right\}\right.\right.\right. \\
& =\left\{v_{m_{1}} \otimes(x, 1), \ldots, v_{m_{n}} \otimes\left(x_{n}, 1\right)\right\}
\end{aligned}
$$

captures the origin with tolerance $t$.

We are almost done. Define $X_{i}=\left\{x_{j}: m_{j}=1\right\}$ for $i \in[r]$. This is an $r$-partition of $X$ and with this partition the set $Y$ is exactly the set $\bar{X}$ that appears in Sarkaria's lemma. As $Y$ captures the origin with tolerance $t$, for every $T \subset X$ of size at most $t, 0 \in \operatorname{conv}(\overline{X \backslash T})$. Sarkaria's lemma implies then that $\bigcap_{1}^{r} \operatorname{conv}\left(X_{i} \backslash T\right) \neq \emptyset$. So $X_{1}, \ldots, X_{r}$ form an $r$-partition of $X$ with tolerance $t$.

Proof of the lemma. Let $G=\left\{g_{1}, \ldots, g_{q}\right\}, q \leq p$. We use induction on $r$. The case $t=0$ is the colourful Carathéodory Theorem 4.1 with $G a_{1}, \ldots, G a_{n}$ as colour classes. Suppose the lemma is true for $t-1$ but false for $t$. Given a vector $\left(h_{1}, \ldots, h_{n}\right) \in G^{n}$ define $h \cdot S=\left\{h_{1} a_{1}, \ldots, h_{n} a_{n}\right\}$. Since the lemma is false for $r$, for every $h \in G^{n}$ there is $T \subset h \cdot S$ with $t$ points so that $h \cdot S \backslash T$ is separated from the origin. So $\operatorname{dist}(0, \operatorname{conv}(h \cdot S \backslash T))>0$.

For a given $h \in G^{n}$ let $D(h)$ denote the minimum of all such distances, so $D(h)>0$. Choose $h^{*} \in G^{*}$ so that $D\left(h^{*}\right)$ is minimal among all the $D(h)$. Let $T^{*}$ be the $t$-element subset of $h^{*} \cdot S$ for which $D\left(h^{*}\right)=\operatorname{dist}\left(0, \operatorname{conv}\left(h^{*} \cdot S \backslash T^{*}\right)\right)$. Write $\triangle=\operatorname{conv}\left(h^{*} \cdot S \backslash T^{*}\right)$, so there is $x \in \triangle$ which realizes this distance. Let $H$ be the hyperplane in $\mathbb{R}^{p}$ that contains $x$ and is orthogonal to $x$. It follows that $x$ is in the convex hull of a set $V \subset\left(h^{*} \cdot S \backslash T^{*}\right) \cap H$ with at most $p$ elements. Write $U=h^{*} \cdot S \backslash V$ and let $H^{-}$be the halfspace bounded by $H$ and containing the origin.

It is easy to see that $U$ is compatible with the action of $G$, and $m=|U| \geq p t+1$. The induction hypothesis yields a vector $k \in G^{|U|}$ such that $k \cdot U$ captures the origin with tolerance $t-1$. Observe that 
for each $b \in U$ there is $g_{i} \in G$ such that $g_{i} b \in H^{-}$. This follows as the set $G b$ captures the origin for every $b \in U$. Consider the sets $\left(g_{1} k\right) \cdot U, \ldots,\left(g_{q} k\right) \cdot U$ written as rows in the matrix below.

$$
\begin{array}{cccc}
g_{1} k_{1} u_{1} & g_{1} k_{2} u_{2} & \ldots & g_{1} k_{m} u_{m} \\
g_{2} k_{1} u_{1} & g_{2} k_{2} u_{2} & \ldots & g_{2} k_{m} u_{m} \\
\vdots & \vdots & \vdots & \vdots \\
g_{q} k_{1} u_{1} & g_{q} k_{2} u_{2} & \ldots & g_{q} k_{m} u_{m}
\end{array}
$$

By the previous observation, every column here contains an element in $H^{-}$. There are $m \geq p t+1$ columns and $q \leq p$ rows. By the pigeonhole principle there is a $g \in G$ such that $(g k) \cdot U$ has at least $t+1$ elements in $H^{-}$.

Next we define a new vector $h \in G^{n}$ by setting $h_{j}=g k_{j}$ if $a_{j} \in U$ and $h_{j}=h_{j}^{*}$ otherwise. We claim that $D(h)<D\left(h^{*}\right)$. Let $T \subset h \cdot S$ be a set of at most $t$ points such that $0 \notin \operatorname{conv}((h \cdot S) \backslash T)$. Now $T$ cannot contain $t-1$ or fewer points from $\left(g h^{*}\right) \cdot U$, because then $h \cdot S \backslash T$ would capture the origin. Thus $T \subset\left(g h^{*}\right) \cdot U$ and then there is a point $a \in H^{-} \cap\left(\left(g h^{*}\right) \cdot U\right)$ that is not in $T$. It follows that conv $(V \cup\{a\})$ is closer to the origin than conv $V$. Thus indeed $D(h)<D\left(h^{*}\right)$ contradicting the minimality of $D\left(h^{*}\right)$.

Open question 9.3. Write $T(d, r, t)$ for the smallest integer such that every set $X \subset \mathbb{R}^{d}$ with $T(d, r, t)$ points has an $r$-partition with tolerance $t$. Theorem 9.1 shows that $T(r, d, t) \leq(r-1)(d+1)(t+1)+1$. What is the exact value of $T(d, r, t)$ ? Even the case $t=1$ is open, the best known lower bound is $\lfloor 5 d / 3\rfloor+3 \leq T(d, r, 1)$, cf [23] and [17].

Some recent results concerning $T(d, r, t)$ for $d \leq 2$ can be found in Mulzer, Stein [20].

Acknowledgments. Thanks are due to Pablo Soberón and the anonymous referees for thorough and critical reading and useful advice. My research was partially supported by ERC Advanced Research Grant no 267165, and by Hungarian National Research Grants K 83767 and NK 78439 .

\section{REFERENCES}

[1] J. Arocha, I. Bárány, J. Bracho, R. Fabila, and L. Montejano. Very colorful theorems. Discrete Comp. Geom., 42 (2009), 142-154.

[2] I. Bárány. A generalization of Carathéodory's theorem. Discrete Math., 40 (1982), 141-152.

[3] I. Bárány, Z. Füredi, L. Lovász. On the number of halving planes. Combinatorica, 10 (1990), 175-185.

[4] I. Bárány, D. G. Larman. A coloured version of Tverberg's theorem. J. London Math. Soc., 45 (1992), 314-320.

[5] I. Bárány, Sh. Onn. Colourful linear programming and its relatives. Math.OR, 22 (1997), 550-567. 
[6] I. Bárány and J. Matoušek. Quadratic lower bound for the number of colourful simplices. SIAM J. on Discrete Math., 21 (2007), 191-198.

[7] I. Bárány, R. Karasev. Notes about the Carathéodory number. Discrete Comp. Geom., 48 (2012), 783-792

[8] A. Björner, L. Lovász, R.T. Živaljević, S.T. Vrećica. Chessboard complexes and matching complexes. J. London Math. Soc., 45 (1944), 25-39.

[9] P. Blagojević, B. Matschke, G. M. Ziegler. Optimal bounds for the colored Tverberg problem. October 2009. (arXiv:math.AT/0910.4987).

[10] C. Carathéodory, Über den Variabilitätsbereich der Fourierschen Konstanten von positiven harmonischen Funktionen. Rend. Circ. Mat. Palermo, 32 (1911), 193-217.

[11] A. Deza, S. Huang, T. Stephen, and T. Terlaky. Colourful simplicial depth. Discrete Comp. Geom., 35 (2006), 597-615.

[12] A. Deza, T. Stephen, F. Xie. More colourful simplices. Discrete Comp. Geom., 45 (2011), 272-278.

[13] A. Deza, F. Meunier, P. Sarrabezolles. A combinatorial approach to colourful simplicial depth. SIAM J. Discrete Math., 28 (2014), 306-322.

[14] W. Fenchel. Über Krümmung und Windung geschlossener Raumkurven. Math. Ann., 101 (1929), 238-252.

[15] A. Holmsen. J. Pach, H. Tverberg. Points surrounding the origin. Combinatorica, 28 (2008), 633-634.

[16] P. Kirchberger. Über Tschebyschefsche Annäherungsmethoden. Math. Ann., 57 (1903), 509-540.

[17] D. G. Larman. On sets projectively equivalent to the vertices of a convex polytope. Bull. London Math. Soc., 4 (1972), 6-12

[18] J. Matoušek. Note on the colored Tverberg theorem. J. Comb. Theory B, 66 (1966), 146-151.

[19] J. W. Milnor. Topology from the Differentiable Viewpoint. The University Press of Virginia, Charlottesville, VA, 1965

[20] W. Mulzer, Y. Stein. Algorithms for tolerated Tverberg partitions. (2013) arXiv:1036.5527.

[21] A. Pór. Diploma thesis. Eötvös University, Budapest, 1998.

[22] J. Radon. Mengen konvexer Körper, die einen gemensamen Punkt erhalten. Math. Ann., 83 (1921), 113-115.

[23] J. L. Ramírez Alfonsín. Lawrence oriented matroids and a problem of McMullen on projective equivalences of polytopes. European J. Comb., 22 (2001), 723-731.

[24] J-P. Roudneff. Partitions of Points into Simplices with k-dimensional Intersection. Part I: The Conic Tverbergs Theorem. Europ. J. Comb., 22 (2001), 733-743.

[25] P. Sarrabezolles. The colourful simplicail depth conjecture. (2014), arXiv:1402.3412.

[26] K. S. Sarkaria. Tverberg's theorem via number fields. Israel J. Math., 79 (1992), $317-320$.

[27] P. Soberón. Equal coefficients and tolerance in coloured Tverberg partitions. arXiv:1204.1202

[28] P. Soberón, R. Strausz. A Generalisation of Tverberg's Theorem. Discrete Comp. Geom., 47 (2012), 455-460.

[29] T. Stephen and H. Thomas. A quadratic lower bound for colourful simplicial depth. J. Comb. Optimization, 16 (2008), 324-327.

[30] H. Tverberg. A generalization of Radon's theorem. J. London Math. Soc., 41 (1966), 123-128. 
[31] H. Tverberg. A generalization of Radon's theorem II. Bull. Austr. Math. Soc., 24 (1981), 321-325.

[32] H. Tverberg, S.T. Vrećica. On generalizations of Radon's theorem and the Ham sandwich theorem. European J. Comb., 14 (1993), 259-264.

[33] G. M. Ziegler. 3N Colored Points in a Plane. Notices of the AMS., 58 (2011) 550-557.

[34] R.T. Živaljević, S.T. Vrećica. The colored Tverberg's problem and complexes of injective functions. J. Comb. Theory A. 61 (1992), 309-318.

[35] M. Yu. Zvagelskii. An elementary proof of Tverberg's theorem. J. Math. Sci. (N. Y.) 161 (2009), 384-387.

\section{IMRE BÁRÁNY}

Rényi Institute of Mathematics Hungarian Academy of Sciences PO Box 127, 1364 Budapest

Hungary

and

Department of Mathematics

University College London

Gower Street, London WC1E 6BT

England

e-mail: barany.imre@renyi.mta.hu 